\title{
Effect of Device Variables on Surface Potential and Threshold Voltagein DG-GNRFET
}

\author{
Baharak Mehrdel ${ }^{*}$, Azlan Abdul Aziz ${ }^{*}$, Mahdiar Hossein Ghadiri** \\ * School of Physics, Universiti Sains Malaysia \\ ** Electrical and Electronic Engineering, Engineering Campus, Universiti Sains Malaysia
}

\begin{tabular}{l} 
Article Info \\
\hline Article history: \\
Received Apr 8, 2015 \\
Revised Jul 7, 2015 \\
Accepted Jul 23, 2015 \\
\hline Keyword: \\
Double gate transistor \\
Graphene nanoribbon transistor \\
Length of saturation \\
Surface potential \\
Threshold voltage
\end{tabular}

\begin{abstract}
In this paper we present four simple analytical threshold voltage model for short- channel and length of saturation velocity region (LVSR) effect that takes into account the built - in potential of the source and drain channel junction, the surface potential and the surface electric field effect on double gate graphene nanoribbon transistors. Four established models for surface potential, lateral electric field, LVSR and threshold voltage are presented. These models are based on the easy analytical solution of the two dimensional potential distribution in the graphene and Poisson equation which can be used to obtain surface potential, lateral electric field, LVSR and threshold voltage. These models give a closed form solution of the surface potential and electrical field distribution as a function of structural parameters and drain bias. Most of analytical outcomes are shown to correlate with outcomes acquired by Matlab simulation and the end model applicability to the published silicon base devices is demonstrated.
\end{abstract}

Copyright (C) 2015 Institute of Advanced Engineering and Science. All rights reserved.

\section{Corresponding Author:}

Baharak Mehrdel,

School of Physics,

Universiti Sains Malaysia,

111800, Pulau Pinang, Malaysia.

Email: baharimehr@gmail.com

\section{INTRODUCTION}

Increasing demand for high speed efficiency in memory and logic applications has created a continuous tendency for smaller device sizes. CMOS technology has shifted to the submicron structure to achieve large density as well as higher efficiency integrated circuits, so the short channel effects can be greater. Among the important factors which determine short-channel effects are the decay of device threshold voltage by using reduced channel length [1]. In order to proceed with this type of scaling factor equality, the channel length regarding silicon MOSFETs as estimated by ITRS will probably need to be scaled to satisfy the needs of next-generation technologies. Nonetheless, there are several question related to the action of silicon devices under excessive scaling. Accordingly, some new material baseddevice has been proposed; for example, nanowire FETs, carbon nanoribbon FET [2-4]. Recent experimental studies have discussed the feasibility of manufacturing graphene nanoribbon transistors $[5,6]$. The majority of scientists have become interested in this area and presented various types of GNR transistor features and applications [7-14]. However, there is an absence of research in modelling those features close to the drain junction, which is known breakdown voltage.

Woo et al and Guo and $\mathrm{Wu}[15,16]$ has created short-channel threshold voltage designs by resolving the two-dimensional Poisson equation. Imam et al [17] designed the threshold voltage by formalizing the two dimensional Poisson formula as a pair of the one dimensional (1D) Poisson formula and two dimensional Laplace formula. For the threshold voltage an exponential function of L was estimated. Lately, Banna [18], applied the quasi two dimensional method and stated that the threshold voltage model 
gave a quite similar dependence on L to thatstated by Imam et al. [17]. In this paper, an easy analytical method for threshold voltage with the short - channel, completely depleted DG-GNRFET can be explained. In addition, an analytical solution is based on the $2 \mathrm{D}$ potential distributions in the graphene film. Suitable boundary conditions can be set for uniform doping in the graphene film. Accordingly, this study presents an uncomplicated design for the surface potential and electrical field distribution of double - gate graphene nanoribbon field effect transistor. During the following section, the surface potential as well as the threshold voltage for short channel of GNR will be defined first. To be able to simplify the analysis, we do not assume interface charges. In addition, carrier accumulation or inversion has been ignored in the graphene film buried oxide. Moreover, we want to explain the behaviour of DG-GNRFET close to the drain junction as well as the breakdown voltage in comparison with the silicon base transistor.

\section{RESEARCH METHOD}

\section{The Proposed Model for LVSR (Surface Potential) and the Short -Channel Solution}

The velocity-saturation-region length of FETs as well as the width of the drain region in which carrier velocity saturation and impact ionization takes place can be more significant variables for short channel devices in nanoscale transistors. The LVSR controls the hot-electron generation, substrate current, the drain breakdown voltage and drain current in the drain region [19-24]. At the FET, when the used drain voltage is greater than the saturation drain voltage, the electric field close to the drain region isgreater than the critical field power which leads to carrier velocity saturation [25]. A standard schematic cross-section from double gate GNRFET is demonstrated in figure 1. Where the oxide thickness of the front and back gates is mentioned by $\mathrm{t}_{\mathrm{OX}}$ with a dielectric constant of $\varepsilon_{O X}$. The $\mathrm{L}, \mathrm{W}, t_{G}$ and $\varepsilon_{G}$ are the length, width, thickness and dielectric constant of the GNR respectively. In general, for analyzing the potential distribution in the graphene, it is necessary to solve the Poisson equation first: [26]

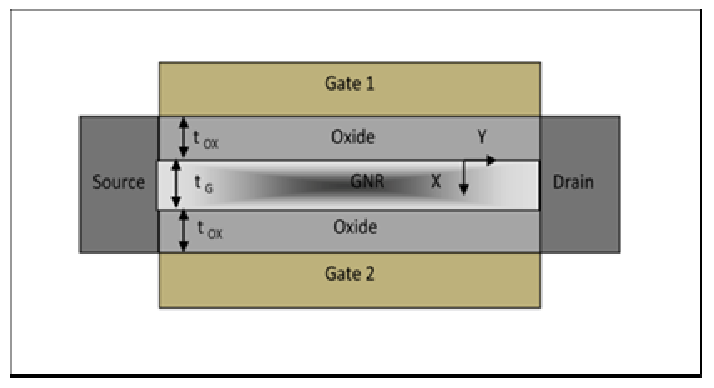

Figure 1. Schematic cross section of a Double Gate GNR FET

$$
\nabla^{2} \Psi(x, y)=\frac{-q N_{d}}{\varepsilon_{G}}, \quad 0 \leq x \leq t_{G}, 0 \leq y \leq L
$$

Where $\Psi(\mathrm{x}, \mathrm{y})$ must be the potential anywhere of $(\mathrm{x}, \mathrm{y})$ throughout the GNR, the electric charge amount is $\mathrm{q}$, the doping concentration of GNR is $\mathrm{N}_{\mathrm{d}}$. In fact, the built- in potential in GNR with a bandgap is not zero. However, in this work, we have included the built-in potential of the source and drain channel junction, for Eq. (1), whereby it is necessary to determine the boundary conditions as, $\Psi(0,0)=V_{\text {bi }}$ and $\Psi(0,, L)=V_{D S}+$ $\mathrm{V}_{\mathrm{bi}}$, the built-in potential and the source-drain voltage are represented by $\mathrm{V}_{\mathrm{bi}}$ and $\mathrm{V}_{\mathrm{DS}}$ respectively. Since the electric flux across the top, down GNR and oxide boundary can be constant, the potential function has to satisfy [27].

$$
\left.\frac{\partial \Psi(\mathrm{x}, \mathrm{y})}{\partial \mathrm{x}}\right|_{\mathrm{x}=0}=\frac{\varepsilon_{O X}}{\varepsilon_{G}} \times \frac{\Psi(0, \mathrm{y})-\mathrm{V}_{\mathrm{g} 1}}{t_{O X}}
$$

and

$$
\left.\frac{\partial \Psi(\mathrm{x}, \mathrm{y})}{\partial \mathrm{x}}\right|_{\mathrm{x}=\mathrm{t}_{\mathrm{G}}}=\frac{\varepsilon_{O X}}{\varepsilon_{G}} \times \frac{\mathrm{v}_{\mathrm{g} 2}-\Psi\left(\mathrm{t}_{\mathrm{G}}, \mathrm{y}\right)}{t_{O X}}
$$


Where $\mathrm{V}_{\mathrm{g} 1}=V_{G S 1}-V_{F B 1}, \mathrm{~V}_{\mathrm{g} 2}=V_{G S 2}-V_{F B 2}, V_{G S 1}$ is the gate-source for front and $V_{G S 2}$ is the gate-source for back flat band voltage. The flat band voltage is $V_{F B}$ for the GNR by using a bandgap $E_{G}=\frac{h V_{F}}{3 w_{G}}[28]$. $V_{F B}=$ $\frac{h V_{F}}{6 q w_{G}}-V_{T} \ln \left(N_{d} / n_{i}\right)$, whereverthe Fermi velocity of the graphene is $V_{F} \sim 10^{6} \mathrm{~m} / \mathrm{s}$. By following the same method analysis as in Refs, [26, 29] to solve Eq. (1), we can decompose $\Psi(x, y)$ into two parts, such that

$$
\Psi(\mathrm{x}, \mathrm{y})=\mathrm{V}(\mathrm{x})+\mathrm{U}(\mathrm{x}, \mathrm{y})
$$

$\mathrm{V}(\mathrm{x})$ can be the one dimension solution from the Poisson equation:

$$
\frac{\partial^{2} V(x)}{\partial x^{2}}=\frac{-q N_{d}}{\varepsilon_{G}}
$$

Eq. (5) is usually used for the long - channel effects. The easiest solution that computes for two dimensional short channel effects is $U(x, y)$ for determining $V(x)$ as provided by Eq. (4), $U(x, y)$ which must fulfil the Laplace equation:

$$
\frac{\partial^{2} \mathrm{U}(\mathrm{x}, \mathrm{y})}{\partial x^{2}}+\frac{\partial^{2} \mathrm{U}(\mathrm{x}, \mathrm{y})}{\partial y^{2}}=0
$$

In Eq. (4), the boundary conditions of $\Psi(\mathrm{x}, \mathrm{y})$ can also be divided into two parts suitable for the solution of Eq. (5) and (6). However, by breaking up Eq. (2) and (3) the boundary conditions are usually displayed as follows:

$$
\left.\frac{\partial \mathrm{V}(\mathrm{x})}{\partial \mathrm{x}}\right|_{\mathrm{x}=0}=\frac{\varepsilon_{O X}}{\varepsilon_{G}} \times \frac{\mathrm{V}(0)-\mathrm{V}_{\mathrm{g} 1}}{t_{O X}}
$$

and

$$
\left.\frac{\partial \mathrm{V}(\mathrm{x})}{\partial \mathrm{x}}\right|_{\mathrm{x}=\mathrm{t}_{\mathrm{G}}}=\frac{\varepsilon_{O X}}{\varepsilon_{G}} \times \frac{\mathrm{v}_{\mathrm{g} 2}-\mathrm{V}\left(\mathrm{t}_{\mathrm{G}}\right)}{t_{O X}}
$$

where before $V_{\mathrm{g} 1}$ and $\mathrm{V}_{\mathrm{g} 2}$, was expressed, it can also be stated $\mathrm{V}(0)=\mathrm{V}_{\mathrm{S} 1}$, which is a front surface potential obtained by resolving Eq. (5), using boundary conditions in Eq. (7), and Eq. (8), and where the device factors can be displayed along with bias conditions like:[27]

$$
V_{S 1}=\frac{C_{G}}{2 C_{G}+C_{O X}}\left[\mathrm{~V}_{\mathrm{g} 2}+\mathrm{V}_{\mathrm{g} 1}\left(\frac{C_{O X}}{C_{G}}+1\right)+\mathrm{qN}_{\mathrm{d}} \mathrm{t}_{\mathrm{G}}\left(\frac{1}{C_{G}}+\frac{1}{C_{O X}}\right)\right]
$$

We can apply the zero gate bias condition, $\mathrm{V}_{\mathrm{GS} 1}=\mathrm{V}_{\mathrm{GS} 2}=0$, so from, Eq. (9) we can write

$$
V_{S 1}=\frac{\mathrm{qN}_{\mathrm{d}}\left(\mathrm{t}_{\mathrm{G}} C_{O X}+\varepsilon_{G}\right)}{C_{O X}\left(2 C_{G}+C_{O X}\right)}
$$

On the other hand, if the device is at gate bias conditions by using $V_{\mathrm{g} 1}=\mathrm{V}_{\mathrm{GS} 1}-\mathrm{V}_{\mathrm{FB} 1}$ from Eq. (9), it can be stated that:

$$
V_{G S 1}=V_{F B 1}+\frac{C_{G}}{C_{G}+C_{O X}}\left[V_{S 1}\left(2+\frac{C_{O X}}{C_{G}}\right)-\mathrm{V}_{\mathrm{g} 2}-\mathrm{qN}_{\mathrm{d}}\left(\frac{\mathrm{t}_{\mathrm{G}} C_{O X}+\varepsilon_{G}}{C_{O X} C_{G}}\right)\right]
$$

In Eq. (6) the boundary conditionscan be stated as; $U(0,0)=V_{b i}-V_{S 1}$ andU $(0, L)=V_{b i}+V_{D S}-V_{S 1}$

$$
\left.\frac{\partial \mathrm{U}(\mathrm{x}, \mathrm{y})}{\partial \mathrm{x}}\right|_{\mathrm{X}=0}=\frac{\varepsilon_{O X}}{\varepsilon_{G}} \times \frac{\mathrm{U}(0, \mathrm{y})}{t_{O X}}
$$

And 


$$
\left.\frac{\partial \mathrm{U}(\mathrm{x}, \mathrm{y})}{\partial \mathrm{x}}\right|_{\mathrm{x}=\mathrm{t}_{\mathrm{G}}}=-\frac{\varepsilon_{\mathrm{OX}}}{\varepsilon_{\mathrm{G}}} \times \frac{\mathrm{U}\left(\mathrm{t}_{\mathrm{G}}, \mathrm{y}\right)}{\mathrm{t}_{\mathrm{OX}}}
$$

with the previous boundary conditions, the results for Eq. (6) can be found from the technique for the differentiation of parameters. The solution obtained at $\mathrm{x}=0$ can be expressed using the series [29]

$$
\mathrm{U}(0, \mathrm{y})=\sum_{\mathrm{n}=1}^{\infty} \mathrm{A}_{\mathrm{n}} \exp \left(\lambda_{\mathrm{n}} \mathrm{y}\right)+\mathrm{B}_{\mathrm{n}} \exp \left(-\lambda_{\mathrm{n}} \mathrm{y}\right)
$$

where

$$
\begin{aligned}
& A_{n}=\frac{\left(V_{b i}+V_{D S}-V_{S 1}\right) \exp \left(-\lambda_{n} L\right)+\left(V_{b i}-V_{S 1}\right) \exp \left(-2 \lambda_{n} L\right)}{1-\exp \left(-2 \lambda_{n} L\right)} \\
& B_{n}=\left(V_{b i}-V_{S 1}\right)-A_{n}
\end{aligned}
$$

In addition, $\lambda_{\mathrm{n}}$ is a parameter that depends on technology, which it can be described as being the solution to the equation:

$$
\mathrm{t}_{\mathrm{G}} \lambda_{\mathrm{n}}=\left(\frac{C_{G}}{2 C_{O X}}\right)\left[\left(\mathrm{t}_{\mathrm{G}} \lambda_{\mathrm{n}}\right)^{2}-\left(\frac{C_{O X}}{C_{G}}\right)^{2}\right] \tan \left(\mathrm{t}_{\mathrm{G}} \lambda_{\mathrm{n}}\right)
$$

The value of $t_{G}$ is small (around $\left.10^{-9}\right)$, so we can approximate tan $\left(t_{G} \lambda_{n}\right)$ tot ${ }_{G} \lambda_{n}$. According to[27], $\lambda_{n}$ is

$$
\lambda_{\mathrm{n}}=\frac{1}{\mathrm{t}_{\mathrm{G}}} \sqrt{1+\frac{2 C_{O X}}{C_{G}}}
$$

We can approach $\Psi(0, y)$ the surface potential distribution of the just initial term $n=1$ from the series in Eq. (14). The main reason is that the results which can be significant for designing the threshold voltage produced from the surface potential are lowest when they happen to behappens close to the centre in the channel $\left(\mathrm{y}_{\min } \cong \mathrm{L} / 2\right)[26]$.

$$
\mathrm{U}(0, \mathrm{y})=\left(V_{b i}+V_{D S}-V_{S 1}\right) \frac{\sinh (\lambda y)}{\sinh (\lambda L)}+\left(V_{b i}-V_{S 1}\right) \frac{\sinh [\lambda(L-y)]}{\sinh (\lambda L)}
$$

Accordingly, for the short channel, the surface potential can be described as

$$
\Psi(0, \mathrm{y})=V_{S 1}+\left(V_{b i}+V_{D S}-V_{S 1}\right) \frac{\sinh (\lambda y)}{\sinh (\lambda L)}+\left(V_{b i}-V_{S 1}\right) \frac{\sinh [\lambda(L-y)]}{\sinh (\lambda L)}
$$

with a minimum given by

$$
\Psi_{\text {min }}=V_{S 1}+\sqrt{\propto_{1} \propto_{2}} \exp \left(-\frac{\lambda \mathrm{L}}{2}\right)
$$

We can determine $\propto_{1}, \propto_{2}$, and the value of $\mathrm{V}_{\mathrm{GS} 1}$ as the short - channel threshold voltage. With the lowest surface potentials $\Psi_{\min }$ equals $2 \emptyset_{F}$ as argued in [26]. Therefore, Eq. (21) can be indicated as:

$$
V_{S 1}=2 \emptyset_{F}-\sqrt{\propto_{1} \propto_{2}} \exp \left(-\frac{\lambda \mathrm{L}}{2}\right)
$$

The value of $V_{G S 1}$ extracted from Eq. (22), by means of $V_{S 1}$ describes the threshold voltage in the shortchannel graphene device. 


$$
V_{G S 1}=\emptyset_{F B 1}+\frac{C_{G}}{C_{G}+C_{O X}}\left[\left(2 \emptyset_{F}-\sqrt{\alpha_{1} \alpha_{2}} \exp \left(-\frac{\lambda \mathrm{L}}{2}\right)\right)\left(2+\frac{C_{O X}}{C_{G}}\right)-\mathrm{V}_{\mathrm{g} 2}-\mathrm{qN}_{\mathrm{d}}\left(\frac{\mathrm{t}_{\mathrm{G}} C_{O X}+\varepsilon_{G}}{C_{O X} C_{G}}\right)\right]
$$

The particular lateral electrical field across the semiconductor surface can be acquired from differentiating Eq. (20).

$$
E_{y}(0, y)=-\frac{\partial \Psi(0, y)}{\partial y}=-\frac{\lambda\left(V_{b i}+V_{D S}-V_{S 1}\right) \cosh (\lambda y)-\lambda\left(V_{b i}-V_{S 1}\right) \cosh [\lambda(L-y)]}{\sinh (\lambda L)}
$$

We take the 1st term $(n=1)$ from the series of Eq. (14), as a surface potential distribution $U(0, y)[23]$. According to [27] from Eq. (14) can be obtained Eq. (25).

$$
\mathrm{U}(0, \mathrm{y})=\left(V_{b i}+V_{D S}-V_{S 1}\right) e^{\lambda(y-L)}+\left(V_{b i}-V_{S 1}\right) e^{-\lambda y}
$$

In Eq. (4), the surface potential along y can be displayed as

$$
\Psi(0, \mathrm{y})=V_{S 1}+\left(V_{b i}+V_{D S}-V_{S 1}\right) e^{\lambda(y-L)}+\left(V_{b i}-V_{S 1}\right) e^{-\lambda y}
$$

Moreover, the lateral electrical field across the channel can be easily obtained by the derivation of Eq. (26), over y.

$$
\mathrm{E}(0, \mathrm{y})=-\lambda\left[\left(V_{b i}+V_{D S}-V_{S 1}\right) e^{\lambda(y-L)}+\left(V_{b i}-V_{S 1}\right) e^{-\lambda y}\right]
$$

Additionally, by taking $\mathrm{y}=\mathrm{L}-L_{D}, \Psi(0, \mathrm{y})=V_{\text {sat }}$. Therefore, for $L_{D}$ we can state that

$$
L_{D}=\mathrm{L}-\frac{1}{\lambda} \ln \left(\frac{\frac{\left(V_{b i}+V_{D S}-V_{S 1}\right)}{e^{\lambda y}} e^{2 \lambda\left(L-L_{D}\right)}-V_{S 1}}{V_{s a t}-V_{S 1}}\right)
$$

which can be solved numerically. In Eq. (28), $V_{\text {sat }}$ is drain saturation voltage and $L_{D}$ is the length of the saturation velocity region. The relationship between the surface potential, electrical field and the length of saturation region with $t_{O X}, t_{G}, V_{D S}$ and $\mathrm{L}$ is shown in the proposed equations.

\section{RESULTS AND DISCUSSION}

In the following section, by using the above procedure, the threshold voltage can be computed in devices of $20 \mathrm{~nm}$ length and several $t_{O X}$ thicknesses. In figure2, the design estimated threshold voltage has been displayed as the function of the channel length and can also be compared with silicon base devices, in ref [30]. Figure 2, shows the surface potential across the channel length in a threshold situation extracted from Eq. (17), as well as Eq. (20) plotted. The proximity between the source and drain in the short channel devices caused the surface potential to vary from $V_{S 1}$. In ref [26] the estimation of the tangent function in $\tan \left(\mathrm{t}_{\mathrm{G}} \lambda_{\mathrm{n}}\right)$ byt $_{\mathrm{G}} \lambda_{\mathrm{n}}$ is based on very thin silicon films. In addition, this estimation was applied because the GNR thickness is very thin if our figureis compared with the threshold voltage in ref [31]. The simulation model used matches all the presumptions in the analysis. Quantum effects do not take into account effects when they begin to play a very important role in GNR thinner than $5 \mathrm{~nm}$. In figure 3, the behavior for the threshold voltage versus channel length $\mathrm{L}$ with different $t_{O X}$ thickness is shown. During this part, the profile of this surface electrical field as well as the potential change displayed and the results of various variables (for instance, drain-source voltage, oxide thickness, channel length and doping concentration on the length of saturation region) can be analyzed near to the drain region. The proposed model can be confirmed by comparing the computed values with the suggested sample, as well as simulations for a Si based device, in ref [30]. 


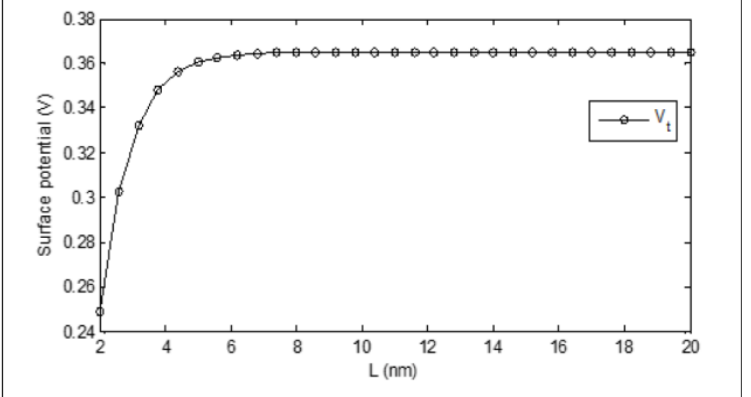

Figure 2. Threshold voltage along the channel length and represents data from the Matlab simulation with $\mathrm{L}=20 \mathrm{~nm}$ from Eq. (17) and Eq. (20)

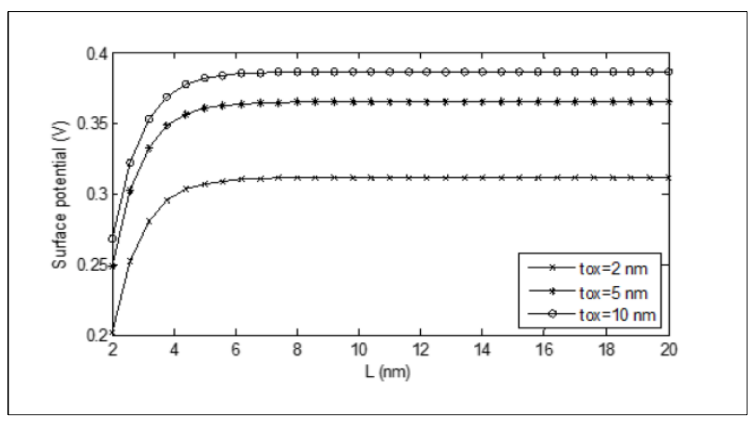

Figure 3. Threshold voltage versus channel length $\mathrm{L}$ for different $t_{\mathrm{OX}}$ thickness of the DG - GNRFET model and represents data from the Matlab simulation with $\mathrm{L}=20 \mathrm{~nm}$ from Eq. (17) and Eq. (20)

A fine settlement can be obtained among simulation outcomes plus the sample with various doping concentrations and oxide thicknesses along with the interval of the drain. The surface potential for $\Psi(0, \mathrm{y})=$ $V_{\text {sat }}$. Figure 4, shows the variance of surface potential across the channel with a variety of drain biases. It can be observed, that as the drain bias is increased the surface potential around the drain side increases if it continues to be constant in the source area, which shows the reliability of our supposed boundary conditions. Figure 5, indicates the change of surface potential across the nanoribbon channel for various oxide thickness with drain bias, $\mathrm{V}_{\mathrm{ds}}=1.5 \mathrm{~V}$ and channel length $\mathrm{L}=15$ and $\mathrm{N}_{\mathrm{d}}=1 \times 10^{15} \mathrm{~cm}^{-3}$. It can be demonstrated thatwhen the oxide thickness decreases the lowest potential close to the source side increases; however, the opposite phenomenon occurs close to the drain due to the fact that the oxide thickness decreases oxide capacitance increases. This boosts the surface charge and the surface potential for the corrected bias conditions. Hence, on the drain side by reducing of the oxide thickness, the oxide capacitance rises together with the off-state current which increases. As a result of this, the potential in the drain side is reduced. From figure6, it can be shown that as the channel lengths are reduced from $L=20 \mathrm{~nm}$ to $\mathrm{L}=15 \mathrm{~nm}$, the surface potential is similar to figure4 for $\mathrm{V}_{\mathrm{ds}}=1.5 \mathrm{~V}$ at each side of the device. However, the lowest potential moves in an upward direction as channel lengths are diminished. This takes place because of the extension of the depletion region below the gate at the surface. Figure 7, shows a strong correlation among the simulation outcomes and samples used in various doping concentrations, with $\mathrm{t}_{\mathrm{OX}}=5 \mathrm{~nm}$ and distances from the drain in double gate GNRFET. When the surface potential model was confirmed the particular LVSR model was also proven to be efficient since it is an ideal method of the surface potential $\Psi(0, y)=V_{S_{1}},[27]$. Furthermore, the surface potential differs in the location of the channel for various peak doping concentrations. It can be stated that when efficient carrier concentration across the channel increases, the surface potential rises. The exact outcomes are as displayed for $\mathrm{V}_{\mathrm{ds}}=1.5 \mathrm{~V}$. Figure 8, shows the field distribution over the nanoribbon surface to the various field oxide layer thicknesses. As explained before,figure5,shows the potential distribution across the channel length for the various field oxide thicknesses. As a result, there is similar effect of the front interface oxide layer thicknesses on the field as well as potential distribution to the silicon based devices. As shown in figure 8, the opposite phenomenon was observed, because the highest field usually occurs in the $\mathrm{p}^{+} \mathrm{n}^{-}$junction interface in which the avalanche breakdown takes place. The field oxide layer thickness effects the breakdown voltage. If it is thick enough tocausea breakdown voltage when the voltage becomes weaker [32].

Figure 9, exhibit the analytical outcomes of the results of the electric field distribution extracted from Eq. (24) for nanoribbon transistors with various doping concentrations $\mathrm{N}_{\mathrm{d}}$. A reasonable evaluation amongst the analytical and numerical final outcomes might usually be discovered. The differences between the two could be as a result of the influence from the space charge region into the $\mathrm{p}^{+}$Base region and $\mathrm{n}^{+}$ Drain diffusion region, which is visible from the field at $\mathrm{x}=0$ and $\mathrm{x}=\mathrm{L}$. Figure 9, shows that the electrical field distribution across the GNR surface for the various doping, compared to the silicon film surface with various doping. It can be clearly shown that there exists two electrical field peaks across the silicon surface, one happens in the $\mathrm{p}^{+} \mathrm{n}^{-}$junction interface and next on the $\mathrm{n}^{+} \mathrm{n}^{-}$junction interface. Close to the drain region demonstrate exactly the same behaviorin theSi based device. As can be seen, the magnitude of the electric field decreases with as the $\mathrm{N}_{d}$ increases [32]. The substrate doping concentration isthe main factor in the optimization of the GNR transistor and defines the amplitude and the position of the maximum peak electric field.

IJECE Vol. 5, No. 5, October 2015 : 1003 - 1011 


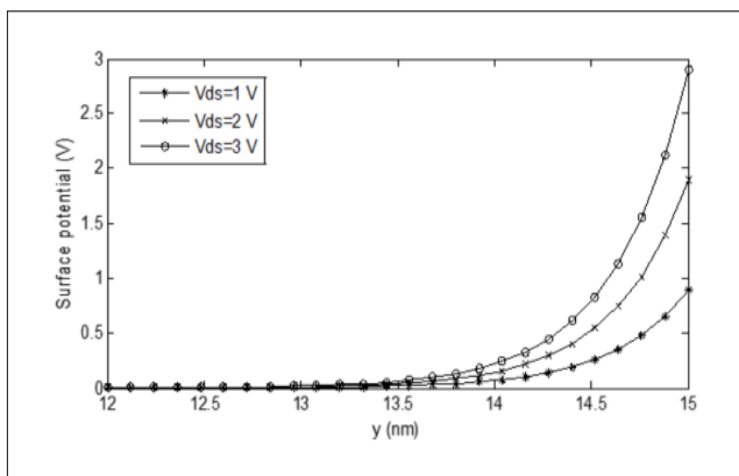

Figure 3. Surface potential versus channel length curves with various drain biases, $\mathrm{L}=15 \mathrm{~nm}$ and oxide thickness $\mathrm{t}_{\mathrm{OX}}=10 \mathrm{~nm}$

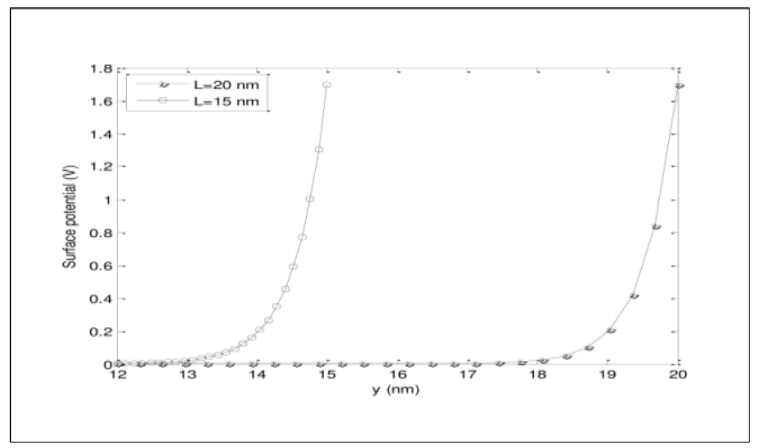

Figure 5. Surface potential curves across the channel with various channel lengths, $\mathrm{t}_{\mathrm{OX}}=5 \mathrm{~nm}, \mathrm{~V}_{\mathrm{ds}}=1.5 \mathrm{~V}$

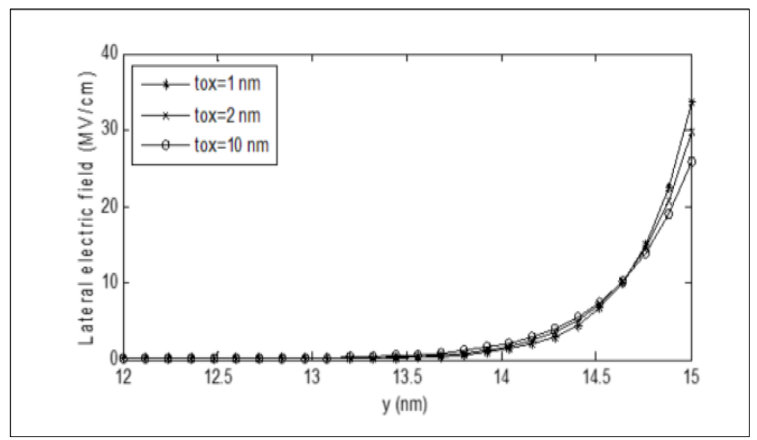

Figure 7. Electrical field distributions across the lateral direction for nanoribbon with various front interface oxide layer thicknesses

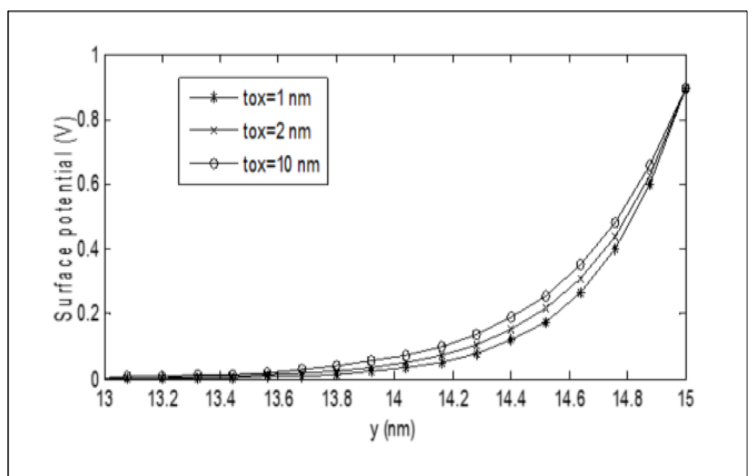

Figure 4. Surface potential curves across the channel with various oxide thicknesses, $L=15 \mathrm{~nm}$ and $V_{\mathrm{ds}}$

$$
=1.5 \mathrm{~V}
$$

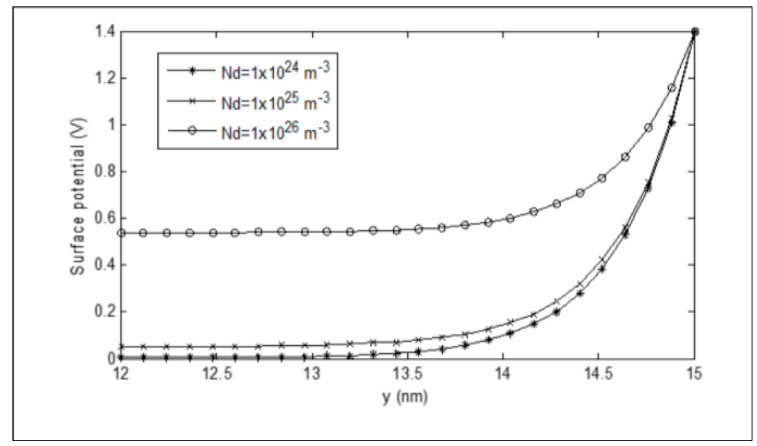

Figure 6. Surface potential curves across the channel with various profile doping concentrations, $\mathrm{L}=15$ $\mathrm{nm}$ and $\mathrm{Vds}=1.5 \mathrm{~V}$

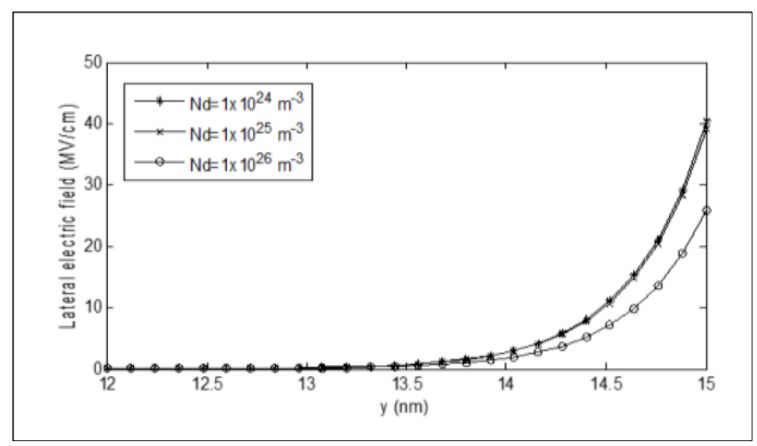

Figure 8. Electrical field distribution across the nanoribbon surface with various doping $\mathrm{N}_{\mathrm{d}}$

\section{CONCLUSION}

From this work, an uncomplicated analytical approach has been found to obtain samples of the threshold voltage for short channel double gate nanoribbon FET which induced the surface potential effects that are usually described and accounted for in our model. Generally, there are analytical models to find the surface potential and electrical field along with LVSR from DG-GNR transistors for the saturation region which was investigated using the recommended model. Additionally, by using the introduced models, the consequences of device variables; for instance nanoribbon thickness, doping concentration and channel 
length was analyzed and compared to the silicon based devices and was proven to beclose to them. As expected, as a result of small-scale geometry for the product, the large lateral electrical field was discovered to be close to the drain junction, becoming a point of trust and reliability of such devices. In addition, the connection among the critical doping concentration around the drift region along with the thickness was described. Most of the analytical outcomes were being shown correlate with the outcomes acquired by Matlab simulation. To increase the breakdown properties for devices, however getting perfect surface field distribution and the critical doping concentration has significant value [32].

\section{ACKNOWLEDGEMENTS}

The authors would like to acknowledge the School of Physic and School of Electrical and Electronic Engineering, UniversitiSains Malaysia, for their support and contribution to this study. Special thanks to Dr. Yoon Tiem Leong for his advice and encouragement

\section{REFERENCES}

[1] Ghadiry M, Nadi M, Bahadorian M, Manaf AABD, Karimi H, Sadeghi H. An analytical approach to calculate effective channel length in graphene nanoribbon field effect transistors. Microelectronics Reliability 2013; 53: 540543.

[2] Deng Y, Fan XY, Han R, Li C. A surface-potential-based model for silicon nanowire junctionless field-effect transistors including interface traps. International Journal of Numerical Modelling: Electronic Networks, Devices and Fields 2014; 27: 869-907.

[3] Zhao WS, Wang G, Hu J, Sun L, Hong H. Performance and stability analysis of monolayer single-walled carbon nanotube interconnects. International Journal of Numerical Modelling: Electronic Networks, Devices and Fields 2014; Early view, DOI: 10.1002/jnm.2027.

[4] Xi L, Xiao-Shi J, Chuai R, Jong-Ho L. A compact 2D potential model for subthreshold characterization of nanoscale fully depleted short channel nanowire MOSFETs. International Journal of Numerical Modelling: Electronic Networks, Devices and Fields 2014; Early view, DOI: 10.1002/jnm.2001.

[5] Liang G, Neophytou N, Lundstrom MS, Nikonov DE. Computational study of double-gate graphene nano-ribbon transistors. Journal of Computational Electronics 2008; 7: 394-397.

[6] Lemme MC, Echtermeyer TJ, Baus M, Kurz H. A graphene field-effect device. Electron Device Letters, IEEE 2007; 28: 282-284.

[7] Karamitaheri H, Neophytou N, Pourfath M, Kosina H. Study of thermal properties of graphene-based structures using the force constant method. Journal of Computational Electronics 2011; 11: 14-21.

[8] Cheli M, Michetti P, Iannaccone G. Model and performance evaluation of field-effect transistors based on epitaxial graphene on SiC. Electron Devices, IEEE Transactions on 2010; 57: 1936-1941.

[9] Ghadiry MH, Manaf AA, Ahmadi MT, Sadeghi H, Senejani MN. Design and analysis of a new carbon nanotube full adder cell. Journal of Nanomaterials 2011; 2011: 36.

[10] Berger C, Song Z, Li X, Wu X, Brown N, Naud CC, Mayou D, Li T, Hass J, Marchenkov AN. Electronic confinement and coherence in patterned epitaxial graphene. Science 2006; 312: 1191-1196.

[11] Ghadiry MH, A'Ain AK, Nadi SM. Design and analysis of a novel low PDP full adder cell. Journal of Circuits, Systems, and Computers 2011; 20: 439-445.

[12] Nikolic BK, Saha KK, Markussen T, Thygesen KS. First-principles quantum transport modeling of thermoelectricity in single-molecule nanojunctions with graphene nanoribbon electrodes. Journal of Computational Electronics 2012; 11: 78-92.

[13] Rahmani M, Ismail R, Ahmadi MT, Rahmani K, Pourasl AH. Trilayer Graphene Nanoribbon Field Effect Transistor Analytical Model. TELKOMNIKA Indonesian Journal of Electrical Engineering 2014; 12: 2530-2535.

[14] Kiani MJ, Ahmadi MT, Rahmani M, Harun FKC. Degeneracy Effect on Carrier transport in Bilayer Graphene Nanoribbon. International Journal of Nano Devices, Sensors and Systems (IJ-Nano) 2013; 2.

[15] Guo JY, Wu CY. A new 2-D analytic threshold-voltage model for fully depleted short-channel SOI MOSFET's. Electron Devices, IEEE Transactions on 1993; 40: 1653-1661.

[16] Woo JCS, Terrill KW, Vasudev PK. Two-dimensional analytic modeling of very thin SOI MOSFETs. Electron Devices, IEEE Transactions on 1990; 37: 1999-2006.

[17] Imam MA, Osman MA, Nintunze N. Modelling the threshold voltage of short-channel silicon-on-insulator MOSFETs. Electronics Letters 1993; 29: 474-475.

[18] Banna SR, Chan PCH, Ko PK, Nguyen CT, Chan M. Threshold voltage model for deep-submicrometer fully depleted SOI MOSFET's. Electron Devices, IEEE Transactions on 1995; 42: 1949-1955.

[19] Baum G, Beneking H. Drift velocity saturation in MOS transistors. Electron Devices, IEEE Transactions on 1970; 17: 481-482.

[20] Gildenblat G, Li X, Wu W, Wang H, Jha A, van Langevelde R, Smit GDJ, Scholten AJ, Klaassen DBM. PSP: An advanced surface-potential-based MOSFET model for circuit simulation. Electron Devices, IEEE Transactions on 2006; 53: 1979-1993. 
[21] Arora ND, Sharma MS. MOSFET substrate current model for circuit simulation. Electron Devices, IEEE Transactions on 1991; 38: 1392-1398.

[22] Fang FF, Fowler AB. Hot electron effects and saturation velocities in silicon inversion layers. Journal of Applied Physics 1970; 41: 1825-1831.

[23] Min B-H, Park C-M, Han M-K. Electrical characteristics of poly-Si TFT's with smooth surface roughness at oxide/poly-Si interface. Electron Devices, IEEE Transactions on 1997; 44: 2036-2038.

[24] Wong H. A physically-based MOS transistor avalanche breakdown model. Electron Devices, IEEE Transactions on 1995; 42: 2197-2202.

[25] Wong H, Poon MC. Approximation of the Length of Velocity Saturation Region in MOSFET's. Electron Devices, IEEE Transactions on 1997; 44: 2033-2036.

[26] Imam MA, Osman MA, Osman AA. Threshold voltage model for deep-submicron fully depleted SOI MOSFETs with back gate substrate induced surface potential effects. Microelectronics Reliability 1999; 39: 487-495.

[27] Ghadiry MH, Nadi S M, Ahmadi MT, Abd Manaf A. A model for length of saturation velocity region in doublegate Graphene nanoribbon transistors. Microelectronics Reliability 2011; 51: 2143-2146.

[28] Zhang Q, Fang T, Xing H, Seabaugh A, Jena D. Graphene nanoribbon tunnel transistors. Electron Device Letters, IEEE 2008; 29(12):1344-1346, DOI 10.1109/LED.2008.2005650.

[29] Harper PG. Introduction to physical mathematics. CUP Archive, 1985.

[30] Baishya S, Mallik A, Sarkar CK. A threshold voltage model for short-channel MOSFETs taking into account the varying depth of channel depletion layers around the source and drain. Microelectronics Reliability 2008; 48: 1722.

[31] Sviličić. B, Jovanović V, Suligoj T. Analytical models of front- and back-gate potential distribution and threshold voltage for recessed source/drain UTB SOI MOSFETs. Solid-state electronics 2009; 53: 540-547.

[32] He J, Zhang X. Quasi-2-D analytical model for the surface field distribution and optimization of RESURF LDMOS transistor. Microelectronics journal 2001; 32: 655-663. 